\title{
Nonlinear two-field models from orbit equation deformations
}

\author{
A. de Souza Dutra ${ }^{1, *}$ and P.E. D. Goulart ${ }^{2, \dagger}$ \\ ${ }^{1}$ UNESP Univ Estadual Paulista-Campus de Guaratinguetá-DFQ, \\ Avenida Doutor Ariberto Pereira Cunha, 33312516-410 Guaratinguetá, SP, Brazil \\ ${ }^{2}$ Instituto de Física Teórica, UNESP Univ Estadual Paulista, Rua Doutor Bento Teobaldo Ferraz, \\ 271 Bloco II-Barra-Funda 01140-070-SP
}

(Received 17 May 2011; published 2 November 2011)

\begin{abstract}
In this work, studying systems of two coupled fields in $(1+1) D$, which present kinklike solutions, we introduce an orbit equation deformation procedure. We apply it in cases which present periodic behavior in the fields potential. We build new periodic potentials using this method.
\end{abstract}

DOI: 10.1103/PhysRevD.84.105001

PACS numbers: 03.65.Ge, 03.65.Ca, 68.65.Fg

\section{INTRODUCTION}

Despite being less common in physical situations, the nonlinear systems are mandatory in order to describe some important systems. For this reason, this subject has attracted an increasing interest in the last years. In fact, the literature these days has plenty of models and applications of solitonic solutions in one and two dimensions [1-16]. For instance, those solutions can be used in order to investigate the formation of domain walls inside domain walls for the case of accelerating universes driven by bulk particles [17]. Furthermore, the case of braneworld scenarios described in five-dimensional space-time with warped geometry involving a single extra dimension, has received considerable attention recently [18,19]. Particularly interesting and connected to the present work are the investigations on this matter through first-order differential equations [19-44]. Another important cosmological issue is the so-called dark energy, which can be examined through Friedmann-Robertson-Walker models described by real scalar fields [26] in the first-order formalism with both one and two scalar fields. In the above cited investigations, and in a great number of other ones, it can be observed that the number of exact nonlinear systems with topological and nontopological configurations for a single scalar field is relatively large in the literature. On the other hand, there are comparatively much fewer examples for two or more coupled scalar fields. Then, any approach capable of increasing the number of models with exact nonlinear solutions should be welcomed.

Some years ago, Bazeia et al. [27] introduced a method capable of obtaining new exact solitonic systems from known ones, which was accomplished in the case of one scalar field models in $1+1$ dimensions. It was later extended to the cases of two interacting scalar fields [28,29]. In the last works, the field deformation is carried out at the level of first-order differential equations of both fields.

\footnotetext{
*Corresponding author. tel. 55-12-3123-2800

dutra@feg.unesp.br

†prieslei@ift.unesp.br
}

However, those methods are a bit intricate, and this fact has stimulated us to seek a simpler method in order to implement the deformation procedure in the case of two coupled scalar fields.

A very important feature, first observed in the case of only one scalar field deformation [27], and which is still present in the more involved situation, is that the deformation approach is capable of generating defects with higher or lesser amplitudes and widths, without changing their topological properties. As a consequence, considering possible condensed matter applications, one is able through this procedure to increase or decrease the mass gap for fermions, allowing a fine-tuning of the gap which may be quite useful for practical purposes.

The Lagrangian density for the case of two coupled scalar fields, which we are going to work with, is given by

$$
\mathcal{L}=\frac{1}{2}\left(\partial_{\mu} \phi\right)^{2}+\frac{1}{2}\left(\partial_{\mu} \theta\right)^{2}-V(\phi, \theta),
$$

whose Euler-Lagrange equations for the static configurations in $1+1$ dimensions are

$$
\begin{gathered}
\frac{d^{2} \phi(x)}{d x^{2}}=W_{\phi} W_{\phi \phi}+W_{\theta} W_{\theta \phi}, \\
\frac{d^{2} \theta(x)}{d x^{2}}=W_{\theta} W_{\theta \theta}+W_{\phi} W_{\phi \theta},
\end{gathered}
$$

where we choose a class of potentials, which can be written in terms of a superpotential $W$, as

$$
V(\phi, \theta)=\frac{1}{2} W_{\phi}^{2}+\frac{1}{2} W_{\theta}^{2},
$$

where $W_{\phi}$ and $W_{\theta}$ stand, respectively, for the differentiation with respect to the fields appearing in the lower index. For this class of systems, one can show that the minimum energy configurations can be obtained from the equivalent system of coupled first-order differential equations [30]

$$
\frac{d \phi}{d x}=W_{\phi}(\phi, \theta), \quad \frac{d \theta}{d x}=W_{\theta}(\phi, \theta) .
$$

Starting from the above differential equations, and constructing the corresponding second-order ones, one gets 


$$
\begin{gathered}
\frac{d^{2} \phi(x)}{d x^{2}}=W_{\phi} W_{\phi \phi}+W_{\theta} W_{\phi \theta}, \\
\frac{d^{2} \theta(x)}{d x^{2}}=W_{\theta} W_{\theta \theta}+W_{\phi} W_{\theta \phi},
\end{gathered}
$$

which becomes identical to those coming from the EulerLagrange Eqs. (2), provided that the superpotential be twice differentiable. In other words, the following restriction shows up

$$
W_{\phi \theta}=W_{\theta \phi} .
$$

This constraint will be of great importance while dealing with the deformation method that will be used to generate the new models. The energy functional whose value at the so-called Bogomol'nyi-Prasad-Sommerfeld configuration corresponding, as we will see below, to the minimal energy can be calculated straightforwardly,

$$
\begin{aligned}
E_{\mathrm{BPS}}= & \frac{1}{2} \int_{-\infty}^{\infty} d x\left[\left(\frac{d \phi}{d x}-W_{\phi}\right)^{2}+\left(\frac{d \theta}{d x}-W_{\theta}\right)^{2}\right. \\
& \left.+2 W_{\theta} \frac{d \theta}{d x}+2 W_{\phi} \frac{d \phi}{d x}\right]
\end{aligned}
$$

From (7), one can see that the minimal energy configurations will come from the solutions obeying the set of first-order differential Eqs. (4). The energy of such configurations is finally given by

$$
E_{\mathrm{BPS}}=\left|W\left(\phi_{i}, \theta_{i}\right)-W\left(\phi_{j}, \theta_{j}\right)\right|,
$$

where $\phi_{i}$ and $\theta_{i}$ are the values of the fields at the $i$ th vacuum state of the model [30].

Instead of applying the usual trial-orbit approach [31], we note that it is possible to write the following equation

$$
\frac{d \phi}{W_{\phi}}=d x=\frac{d \theta}{W_{\theta}},
$$

where the spatial differential element is a kind of invariant. So, one obtains

$$
\frac{d \phi}{d \theta}=\frac{W_{\phi}}{W_{\theta}} .
$$

In general, this last equation is a nonlinear differential equation relating the fields of the model. Now, if one is able to solve it completely, the function $\phi(\theta)$ can be used to eliminate one of the fields, rendering the Eqs. (4) uncoupled. Finally, this uncoupled first-order nonlinear equation can be solved in general, at least numerically.

\section{GENERAL ORBIT EQUATION}

In this section, we illustrate the approach by introducing a new orbit equation, which differs from the one presented in [32,33] by an arbitrary function of the field $\theta$. So one can choose the equation, setting $h(\theta)$, that will satisfy the constraints imposed by the Bogomol'nyi-PrasadSommerfeld method. The orbit equation is

$$
\frac{d \rho}{d \chi}-\frac{\alpha}{\chi}=h(\chi) .
$$

In order to exemplify our procedure, we choose a deformation as follows:

$$
\rho \rightarrow f(\phi), \quad \chi \rightarrow g(\theta),
$$

where $\phi, \theta$ will now represent the deformed fields of the model. Calculating the derivatives of these functions with respect to the fields and introducing the result into (11), one arrives at the following expression of the orbit equation

$$
\frac{d \phi}{d \theta}=\frac{\alpha f(\phi) g_{\theta}(\theta)+g(\theta) g_{\theta}(\theta) h(\theta)}{f_{\phi}(\phi) g(\theta)} .
$$

The superpotentials are then rewritten as

$$
\begin{gathered}
W_{\phi}=\alpha f(\phi) g_{\theta}(\theta)+g(\theta) g_{\theta}(\theta) h(\theta) \\
W_{\theta}=f_{\phi}(\phi) g(\theta) .
\end{gathered}
$$

The functions $f(\phi), g(\theta)$, and $h(\theta)$ are chosen such that the constraint (6) is satisfied. One can check that if we choose $\alpha=1$ and $h(\theta)=\frac{K}{g(\theta) g_{\theta}(\theta)}$, we have

$$
W_{\phi \theta}=f(\phi) g_{\theta \theta}(\theta), \quad W_{\theta \phi}=f_{\phi \phi}(\phi) g(\theta) .
$$

It is important to note that the function $h(\theta)$ was chosen in order to facilitate the choice of the functions $f(\phi)$ and $g(\theta)$ that will satisfy the condition imposed by (6). An inspection of Eqs. (16) leads us to conclude that the functions that must be selected in this example are those which are proportional to their second derivatives, so we pick up functions such as exponentials, sines, cosines, and hyperbolic sines and cosines. This brings the advantage that some of these functions are periodic, and we can build a theory which contains a lot of vacua spread all over the plane $\phi$ versus $\theta$. Thus, we might have many soliton solutions connecting those vacua. Rewriting (13), we have

$$
\frac{d \phi}{d \theta}=\frac{f(\phi) g_{\theta}(\theta)+K}{f_{\phi}(\phi) g(\theta)},
$$

where $K$ is an arbitrary constant. This is a general orbit differential equation. In summary, one can note that the central idea here is that (11) can be solved by using the integrating factor method and (10) can be recast in this form, provided that the constraint (6) holds.

Now, we will show by presenting explicit examples that theories supporting solitons solutions can be built by using this differential equation. The superpotentials are now written as

$$
W_{\phi}=f(\phi) g_{\theta}(\theta)+K, \quad W_{\theta}=f_{\phi}(\phi) g(\theta) .
$$

Integrating the first function with respect to the variable $\phi$ and the second with respect to the variable $\theta$, one can write the superpotential $W(\phi, \theta)$ of the model. 
From now on, we present three cases which, as far as we know, lead to new exact models. In the first example, we shall explore the following transformations on the fields

$$
f(\phi)=\cos (\phi) \quad g(\theta)=\sin (\theta) .
$$

Computing the proper derivatives and replacing in (17), we have

$$
\frac{d \phi}{d \theta}=\frac{\cos \theta \cos \phi+K}{-\sin \theta \sin \phi} .
$$

The superpotentials are then given by

$$
W_{\phi}=\cos \theta \cos \phi+K, \quad W_{\theta}=-\sin \theta \sin \phi .
$$

The differential Eq. (20) can be easily integrated using the well-known method of integrating factor and the result is

$$
\cos \phi=K\left(C_{0} \sin \theta-\cos \theta\right),
$$

where $C_{0}$ is the arbitrary integration constant. This equation is what we mean by orbit function. It represents the fact that one field of the model, in this case the field $\phi$, can be expressed in terms of the other one. It is not always possible to invert the orbit function to obtain $\theta(\phi)$. In order to obtain the function $W(\phi, \theta)$, we integrate the Eqs. (21) respectively with respect to $\phi$ and $\theta$. So, we find out

$$
W(\phi, \theta)=\cos \theta \sin \phi+K \phi .
$$

Since the superpotential is known, the potential can be written as

$$
\begin{aligned}
V(\phi, \theta)= & \frac{1}{2}\left(\cos ^{2} \theta \cos ^{2} \phi+2 K \cos \theta \cos \phi\right. \\
& \left.+\sin ^{2} \theta \sin ^{2} \phi+K^{2}\right) .
\end{aligned}
$$

This potential, which is a kind of two-field sine-Gordon one, is shown in Fig. 1.



FIG. 1 (color online). Two-field sine-Gordon-like potential $V(\phi, \theta)$ for $\alpha=1$ and $K=\frac{\sqrt{2}}{2}$
This potential presents several vacua spread all over the plane $\phi$ versus $\theta$ due to the periodicity of the functions $\sin (\theta)$ and $\cos (\phi)$. With the aid of (22), we can write the second equation in (4) solely in terms of the variable $\theta$ and integrate the result in order to obtain one field $\theta(x)$ as a function of the spatial variable. Once we know how this field depends on the spatial dimension, the field $\phi(x)$ is obtained by replacing the result into (22). The superpotential $W_{\theta}$ is then written as

$$
W_{\theta}=-\sin \theta \sqrt{1-K^{2}\left(C_{0} \sin \theta-\cos \theta\right)^{2}}
$$

The vacua of the potential are obtained when we set $V(\phi, \theta)=0$. They lie at

$$
\begin{aligned}
& \phi=m \pi ; \quad \theta=\arccos (-1)^{m+1} K \\
& \phi=\arccos (-1)^{n+1} K ; \quad \theta=n \pi,
\end{aligned}
$$

where $m, n=0,1,2, \ldots$ We need to replace those values in (22) in order to obtain the dependence of the constant $C_{0}$ in terms of $K$. For even and odd values of $m$, we have $\sin \theta= \pm \sqrt{1-K^{2}}$. Replacing these values in the orbit, we find

$$
C_{0}= \pm \sqrt{\frac{1}{K^{2}}-1}
$$

For our purposes, it is interesting to skip the choice $K=1$ in order to guarantee that $C_{0} \neq 0$ and find critical values, which change the characteristic of the field solutions. For instance, if we choose $K=\frac{1}{\sqrt{2}}$, so $C_{0}= \pm 1$ and, with the aid of (25) and after some rearrangements, we are able to write

$$
\frac{d \theta}{d x}=-\frac{1}{2} \sqrt{(1-\cos 2 \theta)(1 \pm \sin 2 \theta)} .
$$

In spite of being one differential equation of the first order, it is nonlinear and, as far as we know, it can not be solved analytically. So we use numerical methods to obtain a plot of its solution. The solution that represents the other field is also found numerically by using the orbit. The solutions are shown in Fig. 2 for the negative sign in front of the function $\sin 2 \theta$. The field $\theta(x)$ is represented by the left-sided plot and the field $\phi(x)$ is represented by the rightsided plot. We note that the solutions are antikinks that connect the vacuum $(3 \pi / 4,0)$ with the vacuum $(\pi, 3 \pi / 4)$.

The orbit is a straight line. During the numerical integration, we needed to enter with an initial condition for the field $\theta(x)$. This initial condition dictates which vacuum will be connected by the kinks. As we increase or decrease these values, we get different solutions connecting different vacua. All the other solutions are obtained by changing the sign of the term $\sin (2 \theta)$. For lack of space, we are not going to expose here the other solutions, but we present the Fig. 3, which shows the first vacua of the potential and some of their respective orbits. It is noticeable that the orbit 

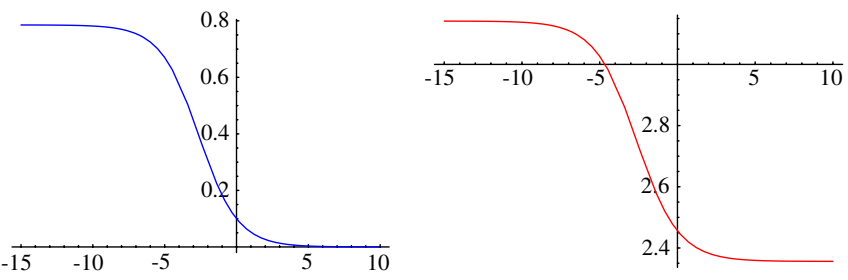

FIG. 2 (color online). Field $\theta(x)$ and $\phi(x)$ for $K=\frac{1}{\sqrt{2}}, C_{0}=$ -1 , and $\theta(0)=0.1$.

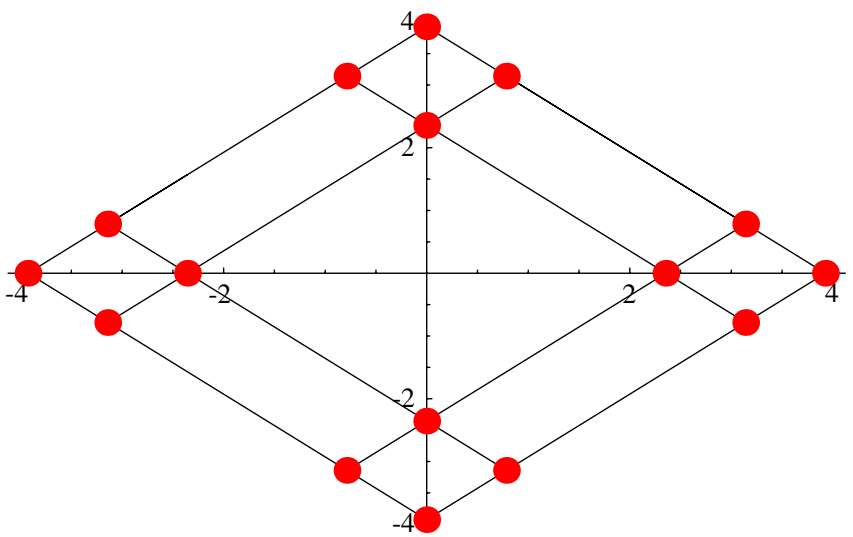

FIG. 3 (color online). Vacua of the potential on the plane $\phi$ versus $\theta$. The orbits that connect the vacua are straight lines.

equation introduced by us generates new potentials supporting kinklike solutions.

As our second example, we construct the potential using the deformation functions

$$
f(\phi)=\cosh (\phi) \quad g(\theta)=\sinh (\theta) .
$$
is

The procedure is the same as before. The orbit equation

$$
\frac{d \phi}{d \theta}=\frac{\cosh \theta \cosh \phi+K}{\sinh \theta \sinh \phi} .
$$

One can easily check that the constraint (6) is respected. The derivatives of the superpotentials are then given by

$$
W_{\phi}=\cosh \theta \cosh \phi+K, \quad W_{\theta}=\sinh \theta \sinh \phi .
$$

Integrating these last two equations, we can obtain the superpotential

$$
W(\phi, \theta)=\cosh \theta \sinh \phi+K \phi
$$

The orbit equation now is

$$
\cosh \phi=K\left(C_{1} \sinh \theta-\cosh \theta\right)
$$

where $C_{1}$ is an arbitrary integration constant. The potential in this case looks like a two-fields version of the sinhGordon model,

$$
\begin{aligned}
V(\phi, \theta)= & \frac{1}{2}\left(\cosh ^{2} \theta \cosh ^{2} \phi+2 K \cosh \theta \cosh \phi\right. \\
& \left.+\sinh ^{2} \theta \sinh ^{2} \phi+K^{2}\right) .
\end{aligned}
$$

Differently from the previous case, we are going to find the vacua before showing a plot of the potential. The vacua lie at

$$
\begin{aligned}
& \theta=0, \quad \phi= \pm \cosh ^{-1}(-K) \\
& \theta= \pm \cosh ^{-1}(-K), \quad \phi=0 .
\end{aligned}
$$

We know that the hyperbolic cosine is an entirely positive function, so we must choose the constant $K$ such that $K<0$. A plot of the potential can now be seen in Fig. 4. In order to find the relation between the two constants of the problem, we replace the values of the fields in the vacua in (34), this way we have the following critical value of the arbitrary integration constant

$$
C_{1}=-\frac{\sqrt{K^{2}-1}}{K} .
$$

As we know the orbit equation, we can write the firstorder differential equation only in terms of the field $\theta$, so

$$
W_{\theta}=\frac{d \theta}{d x}=\sinh \theta \sqrt{K^{2}\left(C_{1} \sinh \theta-\cosh \theta\right)^{2}-1} .
$$

This equation is nonlinear and, as far as we know, there is no general procedure for solving it analytically, but, in order to show the behavior of the solution, we can perform a numerical integration. For this we choose $K=-1.5$, so $C_{1}=\sqrt{5} / 3$. Once we know one field, the other one is found with the aid of the orbit. Figure 5 represents the two fields of the model. The kink represents the field $\theta(x)$ and the antikink represents the field $\phi(x)$



FIG. 4 (color online). Typical profile of the sinh-Gordon-like potential with two interacting scalar fields. 


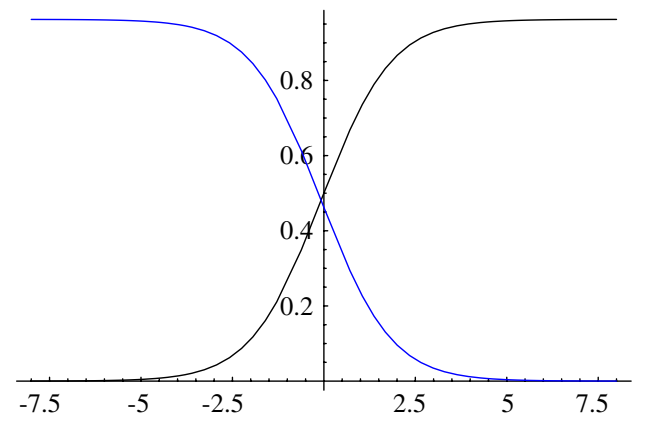

FIG. 5 (color online). Fields of the potential. $\theta(x)$ is represented by the kink (the solution which goes to zero at left) and $\phi(x)$ is represented by the antikink. $K=-1.5$ and $C_{1}=\sqrt{5} / 3$.

In our last example, we show how the method applies for a polynomial transformation of the field $f(\phi)$. The chosen transformations are of the kind

$$
f(\phi)=\phi, \quad g(\theta)=\sin \theta .
$$

We start by putting this transformation into the Eq. (21). So

$$
\frac{d \phi}{d \theta}=\frac{\alpha \phi \cos \theta+K}{\sin \theta}
$$

The superpotentials are written as

$$
W_{\phi}=\alpha \phi \cos \theta+K, \quad W_{\theta}=\sin \theta .
$$

One can easily verify that the constraint imposed by (6) is not satisfied this time. In this case we must, for instance, multiply both equations by an arbitrary function of the field $\phi$ and use (6) to find it. So

$$
W_{\phi}=H(\phi)(\alpha \phi \cos \theta+K), \quad W_{\theta}=H(\phi) \sin \theta .
$$

Computing the derivative of $W_{\phi}$ with respect to $\theta$ and of $W_{\theta}$ with respect to $\phi$, we can write down a differential equation for the function $H(\phi)$, so

$$
\frac{H_{\phi}}{H}=-\alpha \phi
$$

The solution of this equation is

$$
H(\phi)=\exp \left(-\frac{\alpha \phi^{2}}{2}\right) .
$$

Plugging back in (43), we have the superpotentials that satisfy the constraint (6) and can be used to generate a new potential. They are shown below,

$$
\begin{aligned}
& W_{\phi}=\exp \left(-\frac{\alpha \phi^{2}}{2}\right)(\alpha \phi \cos \theta+K) \\
& W_{\theta}=\exp \left(-\frac{\alpha \phi^{2}}{2}\right) \sin \theta
\end{aligned}
$$

Finally we can write the potential using (3) as we have done in the previous examples, so

$$
\begin{aligned}
V(\phi, \theta)= & \frac{1}{2} \exp \left(-\alpha \phi^{2}\right)\left(\alpha^{2} \phi^{2} \cos (\theta)^{2}\right. \\
& \left.+2 \alpha \phi \cos \theta+\sin \theta^{2}+1\right)
\end{aligned}
$$

This kind of "sand dune" potential is represented in Fig. 6, and it looks like a combination of a Liouville potential with a sine-Gordon one. In this case, the vacua are at

$$
\theta=n \pi, \quad \phi=\frac{K}{\alpha}
$$

for odd $n$ and

$$
\theta=n \pi, \quad \phi=-\frac{K}{\alpha}
$$

for even $n$.

The orbit is found solving (41). Notice that in this step it is not necessary to include the function $H(\phi)$ in (41) because the denominator is canceled out with the numerator. The method for solving this equation is the same as used in the previous cases. Different orbits are found choosing different values of the parameter $\alpha$. Its general expression is then

$$
\phi=\sin ^{\alpha} \theta \int \sin ^{-(\alpha+1)} \theta d \theta .
$$

The orbit equation must be smooth in order to guarantee that the potential does not have divergences at any point. This requirement is guaranteed when $\alpha>1$. For sake of simplicity, we choose $\alpha=1$, so the orbit is finally given by

$$
\phi=K\left(C_{0} \sin \theta-\cos \theta\right) .
$$

Replacing this result into (46), we have

$$
W_{\theta}=\exp \left(-\frac{K^{2}\left(C_{0} \sin \theta-\cos \theta\right)^{2}}{2}\right) \sin \theta .
$$

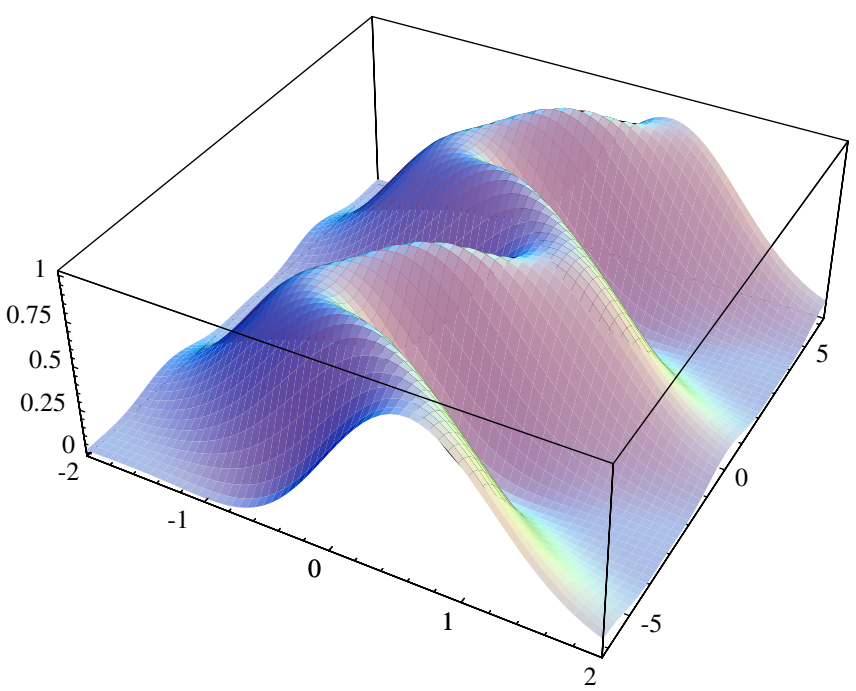

FIG. 6 (color online). " "Sand dune" potential for $\alpha=K=1$. 

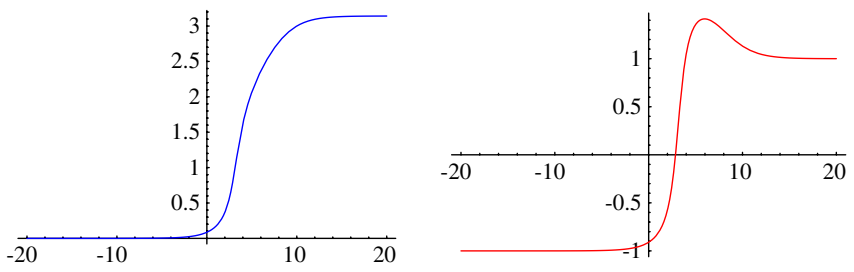

FIG. 7 (color online). Field $\theta(x)$ and $\phi(x)$ for $\alpha=1, K=1$, and $C_{0}=1$.

This equation is again nonlinear, and we solve it numerically in order to obtain the field $\theta(x)$. A plot showing its behavior is shown in Fig. 7. The field $\phi(x)$ is then found following the procedure presented in the previous examples. It is also shown in Fig. 7.

\section{CONCLUSIONS}

In this work, we have introduced an approach to perform the so-called deformation [27] in the case of two interacting scalar fields. In fact, there are other manners which can be used [28,29]. However, as it can be verified, those other approaches are quite involved, and this fact has stimulated us to look for a further, and simpler, method in order to develop the deformation of a given nonlinear model with known solitonic solutions, in order to generate novel exact models. In fact, the search for nonlinear models with exact solution is a nontrivial task, and the introduction of new approaches capable of dealing with this problem is certainly welcomed. Here we did the deformation directly on the equation which defines the orbits relating the two interacting fields. Furthermore, since the examples analyzed in this work are of an oscillating nature in the coupled scalar fields, we can think of applications in condensed matter systems presenting periodic structure and even in the realm of braneworld cosmologies, which has been attracting a lot of interest in recent years. Those questions are of current interest. In fact, in the works dealing with braneworld scenarios using exactly solvable models, one can observe that the field potential is given in terms of a superpotential through

$$
V(\phi)=\frac{1}{2}\left(\frac{d W(\phi)}{d \phi}\right)^{2}-\frac{4}{3} W(\phi)^{2} .
$$

This form of the field potential leads unavoidably to potentials which are unbounded from below for the most part of the systems analyzed in the literature, where $W(\phi)$ is a polynomial in the scalar field. The only exception up to our knowledge is a model studied by Gremm [23], where the superpotentiial is of an oscillatory nature. This feature prevents it from being unbounded [34]. On the other hand, there are reasons to introduce models where the superpotential depends on two scalar fields [35-37] as, for instance, the problem of particle localization on the branes [37]. Thus, we realize that the above introduced model, whose potential is oscillating in the fields, may be interesting in order to study the problem of branes with a bounded potential.

\section{ACKNOWLEDGMENTS}

The authors are grateful to $\mathrm{CNPq}$ and FAPESP for partial financial support.
[1] C. K. Chiang, C. R. Fincher, Jr., Y. W. Park, A. J. Heeger, H. Shirakawa, E. J. Louis, S.C. Gau, and A.G. MacDiarmid, Phys. Rev. Lett. 39, 1098 (1977).

[2] H. W. Fink and C. Schönenberger, Nature (London) 398, 407 (1999).

[3] E. Witten, Nucl. Phys. B249, 557 (1985).

[4] J.R. Morris, Phys. Rev. D 49, 1105 (1994); 51, 697 (1995).

[5] J. D. Edelstein, M. L. Trobo, F. A. Brito, and D. Bazeia, Phys. Rev. D 57, 7561 (1998).

[6] G. J. Cheetham, E. J. Copeland, T. S. Evans, and R. J. Rivers, Phys. Rev. D 47, 5316 (1993).

[7] G. Dvali and M. Shifman, Phys. Lett. B 396, 64 (1997); 407, 452(E) (1997).

[8] A. B. Adib and C. A. S. Almeida, Phys. Rev. E 64, 037701 (2001).

[9] D. Bazeia, Braz. J. Phys. 32, 869 (2002).

[10] M. Eto and N. Sakai, Phys. Rev. D 68, 125001 (2003).

[11] A. A. Andrianov, V. A. Andrianov, P. Giacconi, and R. Soldati, J. High Energy Phys. 7 (2003) 063.
[12] P. Sutcliffe, Phys. Rev. D 68, 085004 (2003).

[13] J. A. R. Cembranos, A. Dobado, and A. L. Maroto, Phys. Rev. D 70, 096001 (2004).

[14] T. House and A. Lukas, Phys. Rev. D 71, 046006 (2005).

[15] D. Bazeia, M. J. dos Santos, and R. F. Ribeiro, Phys. Lett. A 208, 84 (1995).

[16] D. Bazeia and F. A. Brito, Phys. Rev. Lett. 84, 1094 (2000).

[17] F. A. Brito, F. F. Cruz, and J. F. N. Oliveira, Phys. Rev. D 71, 083516 (2005).

[18] L. Randall and R. Sundrum, Phys. Rev. Lett. 83, 3370 (1999); 83, 4690 (1999).

[19] V. I. Afonso, D. Bazeia, and L. Losano, Phys. Lett. B 634, 526 (2006).

[20] M. Cvetic, S. Grifies, and S. Rey, Nucl. Phys. B381, 301 (1992).

[21] K. Skenders and P. K. Towsend, Phys. Lett. B 468, 46 (1999).

[22] O. DeWolfe, D. Z. Freedman, S. S. Gubser, and A. Karsh, Phys. Rev. D 62, 046008 (2000). 
[23] M. Gremm, Phys. Lett. B 478, 434 (2000).

[24] R. Kallosh and A. Linde, J. High Energy Phys. 02 (2000) 005 .

[25] E. E. Flanagan, S. H. Henry Tye, and I. Wisserman, Phys. Lett. B 522, 155 (2001).

[26] D. Bazeia, C. B. Gomes, L. Losano, and R. Menezes, Phys. Lett. B 633, 415 (2006).

[27] D. Bazeia, L. Losano, and J. M. C. Malbouisson, Phys. Rev. D 66, 101701(R) (2002).

[28] A. de Souza Dutra, arXiv:0705.3237.

[29] V. I, Afonso, D. Bazeia, M. A. Gonzales Leon, L. Losano, and J. Mateos Guilarte, Phys. Rev. D 76, 025010 (2007).

[30] D. Bazeia and F. A. Brito, Phys. Rev. D 61, 105019 (2000), and references therein.

[31] R. Rajaraman, Solitons and Instantons (North Holland, Amsterdam, 1982); Phys. Rev. Lett. 42, 200 (1979).

[32] A. de Souza Dutra, Phys. Lett. B 626, 249 (2005).

[33] A. de Souza Dutra and A. C. Amaro de Faria, Jr., Phys. Lett. B 642, 274 (2006).

[34] A. E. R. Chumbes, A. E. O. Vasquez, and M. B. Hott, Phys. Rev. D 83, 105010 (2011).
[35] A. Campos, Phys. Rev. Lett. 88, 141602 (2002).

[36] A. de Souza Dutra, A. C. Amaro de Faria, Jr., and M. Hott, Phys. Rev. D 78, 043526 (2008), and references therein.

[37] R. A. C. Correa, A. de Souza Dutra, and M. B. Hott, Classical Quantum Gravity 28, 155012 (2011).

[38] M. K. Prasad and C. M. Sommerfield, Phys. Rev. Lett. 35, 760 (1975); E. B. Bolgomonyi, Sov. J. Nucl. Phys. 24, 861 (1976).

[39] A. de Souza Dutra and A. C. Amaro de Faria, Jr., Czech. J. Phys. 54, 1229 (2004).

[40] D. Bazeia, L. Losano, and C. Wotzasek, Phys. Rev. D 66, 105025 (2002).

[41] A. de Souza Dutra and M. Hott, Phys. Rev. A 44, 4122 (1991).

[42] A. de Souza Dutra and A. C. Amaro de Faria, Jr., Phys. Lett. B 642, 274 (2006).

[43] D. Bazeia, A. S. Inácio da Silva, and L. Losano, Int. J. Mod. Phys. A 19, 575 (2004).

[44] T. Vachaspati, Kinks and Domain Walls: An Introduction to Classical and Quantum Solitons (Cambridge University Press, Cambridge, England, 2006). 